\title{
Recommendations for optimizing acromioclavicular fixation success with the tight rope device
}

\begin{abstract}
Purpose: To determine if the TightRope device (Arthrex Naples, FL) for fixation of acromioclavicular (AC) separations can create an environment for biologic healing of the coracoclavicular (CC) ligaments.
\end{abstract}

Methods: Twenty-five male subjects, sustained Grade V AC separations. All patients had AC reconstruction with the TightRope device (Arthrex, Naples, FL). The time from the injury to surgical fixation averaged 13 days.

Results: Twenty-one of 25 patients had an anatomic reduction of their AC joint. Four were reduced to a near-anatomic (within $2 \mathrm{~mm}$ ) reduction. At final follow-up, four (16\%) patients maintained their anatomic reduction. These four patients underwent surgery at an average of 11 days after the date of injury and had an average age of 25 years. Thirteen $(52 \%)$ patients displaced to a grade II level (Rockwood classification). These patients averaged 10 days from the date of injury to the date of surgery and had an average age of 39 years. Eight (32\%) of the 25 had displaced back to a grade III level (Rockwood classification). These patients averaged 18 days from the date of injury until date of surgery and had an average age of 35 years. All 25 patients described a subjective improvement regardless of displacement.

Conclusion: There is a high rate of loss of reduction when using the TightRope (Arthrex, Naples, FL) device. When this device is employed, it should be done as quickly as possible after injury. When the time from injury to surgery exceeds 11 days, loss of reduction seems to be greater. There may be a correlation between older age and a more significant loss of reduction regardless of the acuity of fixation.

Level of evidence: IV.
Volume 8 Issue I - 2017

\author{
Lauren E Simonian,' Taylor L Simonian,' \\ Peter T Simonian ${ }^{1,2}$ \\ 'imonian Sports Medicine Clinic, USA \\ ${ }^{2}$ Clinical Associate Professor, Department of Orthopaedic \\ Surgery and Sports Medicine, University of Washington, US
}

Correspondence: Peter T Simonian, Simonian Sports Medicine Clinic, 729 N Medical Center Drive West, Suite 10I, Clovis, CA 936II, USA, Email ptsimonian@earthlink.net

Received: April 10, 2017 | Published: April 21, 2017

\section{Introduction}

Treatment of acute acromioclavicular (AC) disruptions is controversial. ${ }^{1-2}$ Both operative and non-operative treatments can be acceptable. High grade AC injuries, with complete disruption of the coracoclavicular (CC) ligaments, have been those considered for surgical intervention. ${ }^{3-4} \mathrm{~A}$ variety of surgical procedures have been proposed over the years..$^{5-11}$ Complications after AC surgery are relatively common with the most common being loss of reduction. Other problems include hardware failure, ${ }^{12-14}$ fracture of the clavicle or coracoid, ${ }^{15}$ and heterotopic ossification.

With the advent of advanced arthroscopic techniques, some fixation devices have been appealing from the perspective of ease of application and minimal invasiveness. The TightRope device (Arthex, Naples, FL) is one such device. It can be used to reduce an AC separation. It can be applied efficiently and safely in an arthroscopic assisted fashion. Other advantages are that it is a non-rigid device and does not require removal. There have been reported high rates of failure with this device. ${ }^{16-17}$

We theorize that the sooner the CC ligaments are approximated after injury, through anatomic reduction of the $\mathrm{AC}$ joint, the better chance of healing and less chance of loss of reduction.

\section{Material and methods}

Twenty-five male subjects, with a mean age of 35 years (1764 years), sustained Grade V (Rockwood classification, (3)) AC separations. All patients had AC reconstruction with the TightRope device (Arthrex, Naples, FL). The time from the injury to surgical fixation averaged 13 days (5-38 days).
All patients had radiographs at the time of injury, intraoperative, 6 weeks, 12 weeks, 18 weeks and 24 weeks postoperative. All radiographs were evaluated for reduction of the AC joint and intact fixation.

\section{Surgical technique}

All patients underwent arthoscopic assisted AC joint reconstruction. Patients were placed in the beach-chair position. From the posterior portal, the base of the coracoid was visualized with a 70 degree arthroscope. A vector guide with a guidewire protection stop, inserted through an anterior portal, was placed at the base of the coracoid. A guidewire was inserted through both cortices of the clavicle and the coracoid under arthroscopic visualization. Great care was taken to make certain that the wire was centered anteriorposterior on the clavicle. The wire was placed approximately 2-4 $\mathrm{cm}$ medial to the $\mathrm{AC}$ joint. The wire was over-drilled with a $4.0 \mathrm{~mm}$ drill under arthroscopic visualization. The wire was protected with a curette to prevent advancement during over-drilling. A flexible suture passing wire was placed through the cannulation of the drill. The drill removed and the tightrope pulled into position. The coracoid button was flipped under arthroscopic visualization (Figure 1). The clavicle was reduced manually by a surgical assistant while the top button was fixed into position on the superior surface of the clavicle. It was secured with a minimum of 5 square knots.

No patient in this group underwent distal clavicle excision or placement of any biologic graft.

\section{Postoperative protocol}

Pre post operative technique:All patients were immobilized in a sling, except for gentle, self-directed, range of motion exercises 
for six weeks. Slings were discontinued at six weeks and range of motion exercises progressed through both self-directed and formal physical therapy regimens. Patients were instructed not to lift, pull, or push more than five pounds during this 6-12 week postoperative period. Patients were advanced with their weight bearing activities in a graduated fashion during the next 12 weeks and allowed to return to full activity without restriction at six months postoperative.

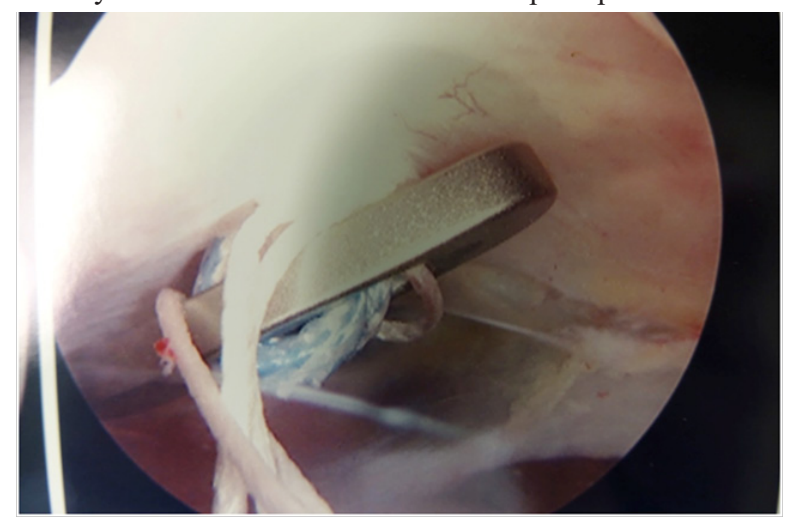

Figure I Arthroscopic (70 degree) view of flipped button under coracoid.

\section{Results}

Twenty-one of 25 patients had an anatomic reduction of their AC joint (Figure 2). Four were reduced to a near-anatomic (within 2mm) reduction. There were no intraoperative complications. There were no fractures of the coracoid or the clavicle.

At final follow-up, four (16\%) patients maintained their anatomic reduction. These four patients underwent surgery at $6,8,13,16$ days (average 11 days) after the date of injury. The ages of this group were $17,19,33$, and 33 . The remaining 21 patients had some degree of loss of reduction. The loss of reduction based on the $\mathrm{CC}$ distance averaged $7.8 \mathrm{~mm}$ with a range of $5-15 \mathrm{~mm}$.

At final follow-up, no patient displaced to the preoperative state. Thirteen (52\%) patients displaced to a grade II level (Rockwood classification $^{3}$ ). These patients averaged 10 days (4-16 days) from the date of injury to the date of surgery. (Figure $3 \&$ Table 1) Eight $(32 \%)$ of the 25 had displaced back to a grade III level (Rockwood classification ${ }^{3}$ ). These patients averaged 18 days (6-38 days) from the date of injury until date of surgery (Figure $4 \&$ Table 1 ).

Table I Patients final follow-up

\begin{tabular}{|c|c|c|c|}
\hline & $\begin{array}{l}\text { No } \\
\text { Displacement }\end{array}$ & $\begin{array}{l}\text { Grade II } \\
\text { Displacement }\end{array}$ & $\begin{array}{l}\text { Grade III } \\
\text { Displacement }\end{array}$ \\
\hline Number of Patients (\%) & $4(16 \%)$ & $13(52 \%)$ & $8(32 \%)$ \\
\hline $\begin{array}{l}\text { Mean time from Injury to } \\
\text { Surgery }\end{array}$ & II days & 10 days & 18 days \\
\hline Average Age & 25 years & 39 years & 33 years \\
\hline
\end{tabular}

Patients of younger age seem to have better results when comparing the no displacement group to those that displaced to a grade II. Surgery in both groups was performed within 11 days of injury. The no displacement group had an average age of 25 years while the grade II displacement group had an average age of 39 years (Table 1). All 25 patients described a subjective improvement regardless of displacement.

\section{Discussion}

The purpose of this study was to determine if a non-biologic AC fixation technique performed within a short time period of injury could allow for healing of the acutely torn CC ligaments.

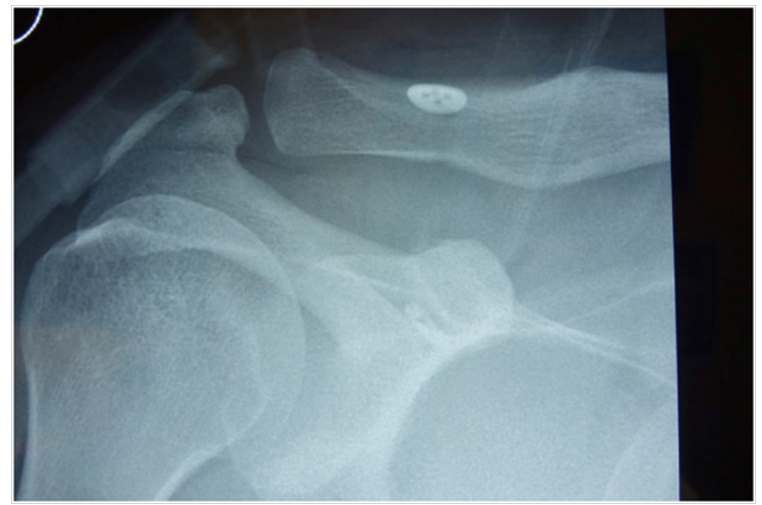

Figure 2 Intra-operative radiograph of anatomic reduction of $A C$ separation with the Tight-rope device (Arthrex, Naples, FL).

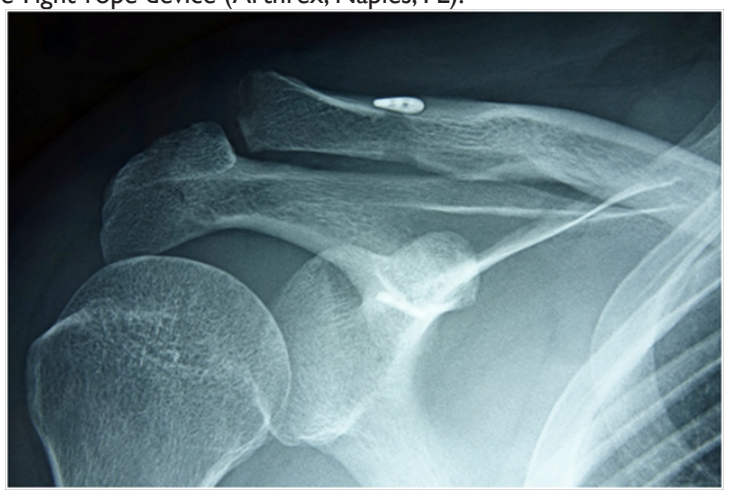

Figure 3 Grade II loss of reduction, 24 weeks after surgery.

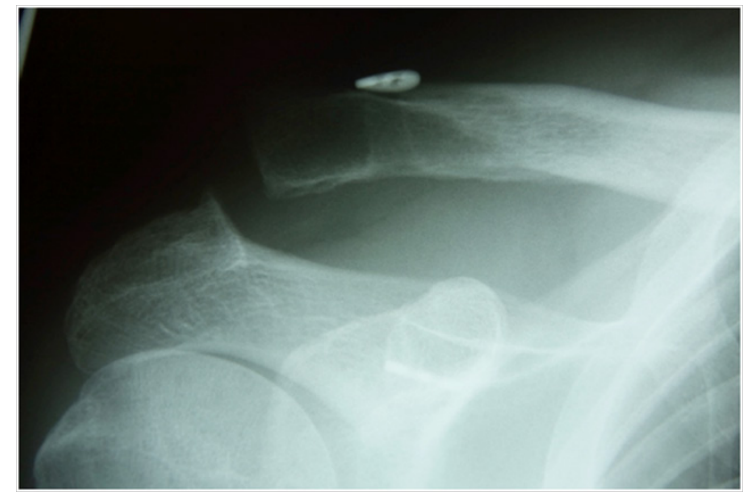

Figure 4 Grade III loss of reduction, 24 weeks after surgery.

Although the numbers of this study are limited, it appears that there is a trend toward less displacement if the surgical repair is performed within 11 days of injury. It appears that failure rates are more significant when surgery is performed more than 18 days after injury.

Patients of younger age may have better results when comparing the no displacement group to those that displaced to a grade II. Surgery in both groups was performed within 11 days of injury. The no displacement group had an average age of 25 years while the grade II displacement group had an average age of 39 years.

A proposed mechanism for failure of the device in the cases that allowed for loss of reduction includes abrasion and disruption of the suture or knot slippage. ${ }^{16} \mathrm{We}$ are uncertain of the mode of failure since none of the devices showed any signs of migration. If the knot slipped or a suture failed, the remaining suture loops may have kept the implants in place and prevented migration of either button. 
The subjective improvement in all subjects seen in this study, regardless of reduction loss, has been reported by others. ${ }^{17}$

This study has limitations. It is a retrospective review with a limited number of patients and follow-up. However the results can help to define some guidelines for more successful application of the TightRope device (Arthrex, Naples, FL) that is currently being used by orthopaedic surgeons for AC separation fixation.

If the Tight-rope device (Arthrex, Naples, FL) or similar is employed, patients should be counseled on the high rate of some recurrence of displacement. The device should be employed as soon as possible after injury; preferably within 11 days of injury. Older patients may have a higher rate of displacement.

Thiel et al. ${ }^{17}$ proposed using two TightRope devices (Arthrex, Naples, FL) to minimize reduction loss. This approach could potentially increase the risk of fracture; especially if biologic healing does not occur.

\section{Conclusion}

The TightRope device (Arthrex, Naples, FL) for fixation of AC separation must create an environment for biologic healing of the CC ligaments to be successful. There is a high rate of loss of reduction when using this device. Patients should be counseled preoperatively of this fact. When this device is employed, it should be done as quickly as possible after injury. When the time from injury to surgery exceeds 11 days, loss of reduction seems to be greater. There may be a correlation between older age and a more significant loss of reduction regardless of the acuity of fixation.

\section{Acknowledgments}

None.

\section{Conflicts of interest}

None.

\section{References}

1. Schlegel TF, Burks RT, Marcus RL. A prospective evaluation of untreated acute grade III acromioclavicular separations. Am J Sports Med. 2001;29(6):699-703.

2. Press J, Zuckerman JD, Gallagher M, et al. Treatment of grade III acromioclavicular separations. Operative versus nonoperative management. Bull Hosp Jt Dis. 1997;56(2):77-83.
3. Williams GR, Nguyen VD, Rockwood CA. Classification and radiographic analysis of acromioclavicular disloca- tions. Appl Radiol. 1989;18:29-34.

4. Urist MR. Complete dislocation of the acromioclavicular joint: the nature of the traumatic lesion and effective methods of treatment with an analysis of 41 cases. J Bone Joint Surg. 1946;28(4):813-837.

5. Phillips AM, Smart C, Groom AFG. Acromioclavicular dislocation: conservative or surgical therapy. Clin Orthop Relat Res. 1998;353:10-17.

6. Boswoth BM. Acromioclavicular separations: new method of repair. Surg Gynecol Obstet. 1941;73:866-871.

7. Larsen E, Petersen V. Operative treatment of chronic acromioclavicular dislocation. Injury. 1987;18(1):55-56.

8. Weaver JK, Dunn HK. Treatment of acromioclavicular injuries, especially complete acromioclavicular separation. J Bone Joint Surg Am. 1972;54(6):1187-1194.

9. Urist MR. Complete dislocation of the acromioclavicular joint. $J$ Bone Joint Surg. 1963;45A:1750-1753.

10. Mumford EB. Acromioclavicular dislocation: A New Operative Treatment. J Bone Joint Surg. 1941;23(4):799-802.

11. Berson BL, Gilbert MS, Green S. Acromioclavicular dislocations: treatment by transfer of the conjoined tendon and distal end of the coracoid process to the clavicle. Clin Orthop. 1978;135:157-164.

12. Norrell H, Llewellyn RC. Migration of a threaded steinman pin from the acromioclavicular joint to the spinal canal: a case report. J Bone Joint Surg. 1965;47A:1024-1026.

13. Lindsey RW, Gutowski WT. The migration of a broken pin following fixation of the acromioclavicular joint: a case report and review of literature. Orthopaedics. 1986;9(3):413-416.

14. Lyons FA, Rockwood CA. Migration of pins used in operations on the shoulder. J Bone Joint Surg Am. 1990;72(8):1262-1267.

15. Ball SV, Sankey A, Cobiella C. Clavicle fracture following tight rope fixation of acromioclavicular joint dislocation. Injury. 2007;38:430-432.

16. Lim YW, Sood A, van Riet RP, et al. Acromioclavicular joint reduction, repair and reconstruction using metallic buttons - early results and complications. Tech in Should \& Elbow Surg. 2007;8(4):213-221.

17. Thiel E, Mutnal A, Gilot GJ. Surgical outcome following arthroscopic fixation of acromioclavicular joint disruption with the Tight Rope device. Orthop. 2011;34(7):e267-e274. 